
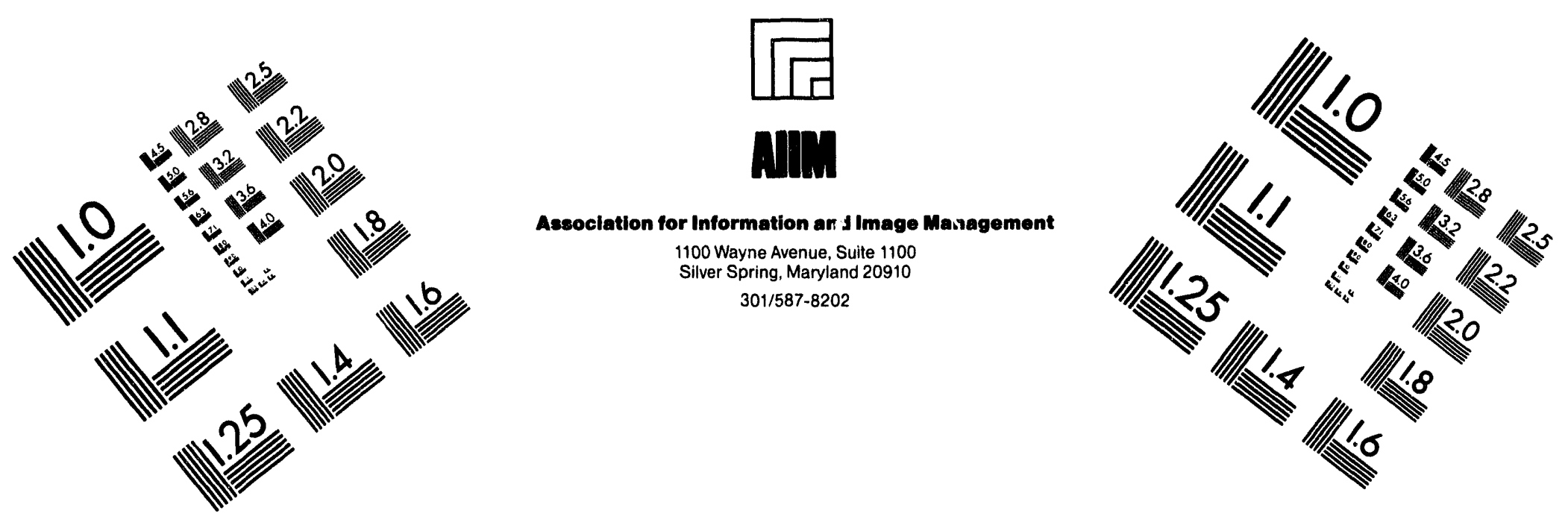

Centimeter

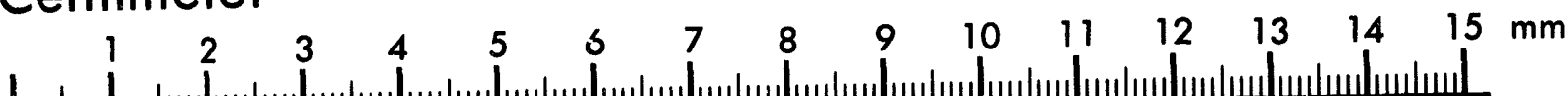

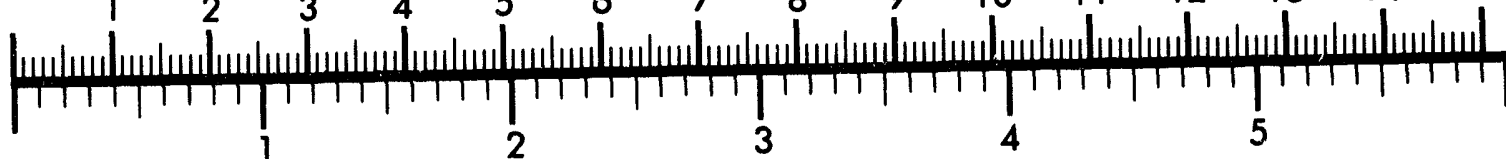
Inches
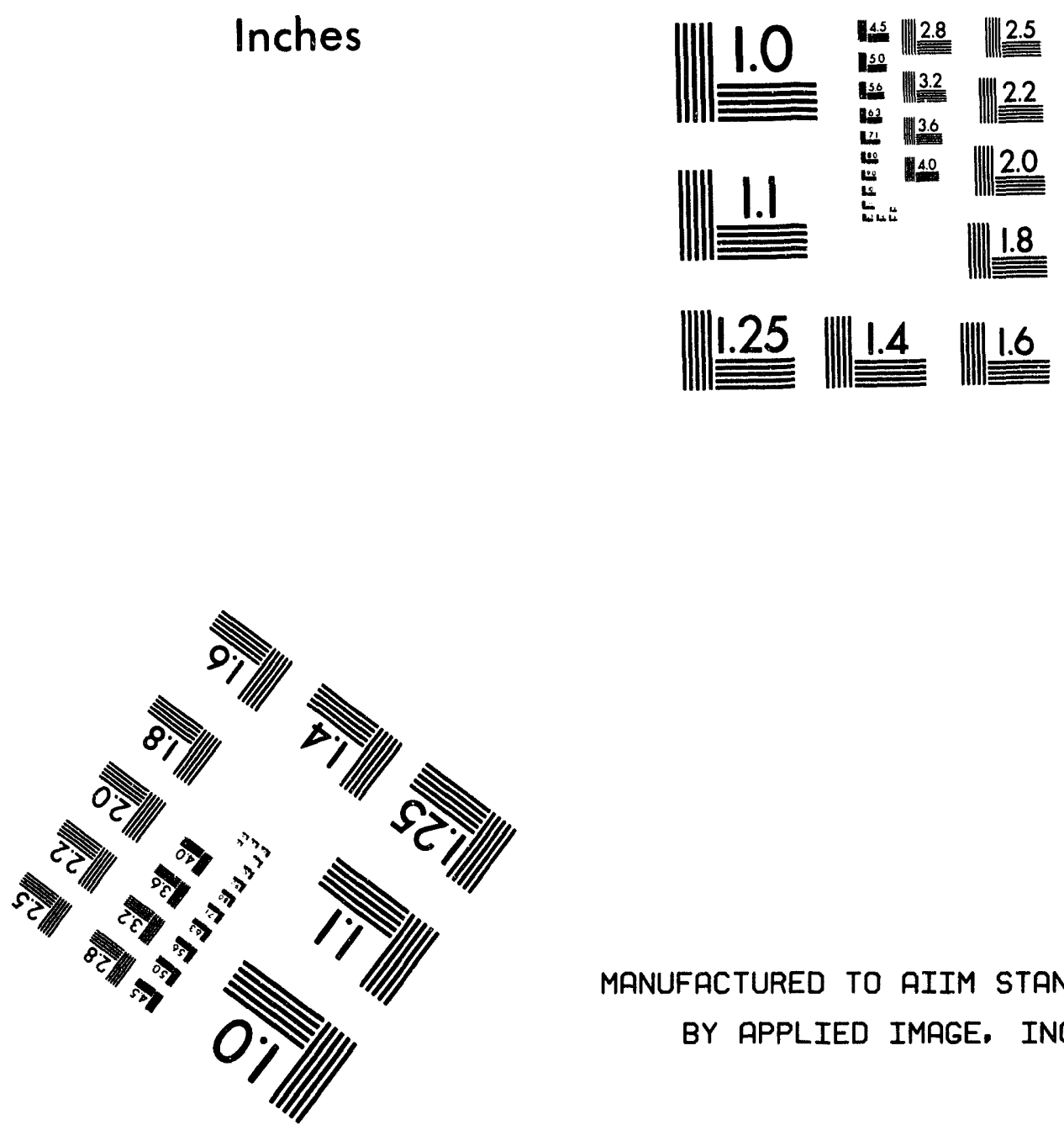

MANUFACTURED TO AIIM STANDARDS

BY APPLIED IMAGE, INC.

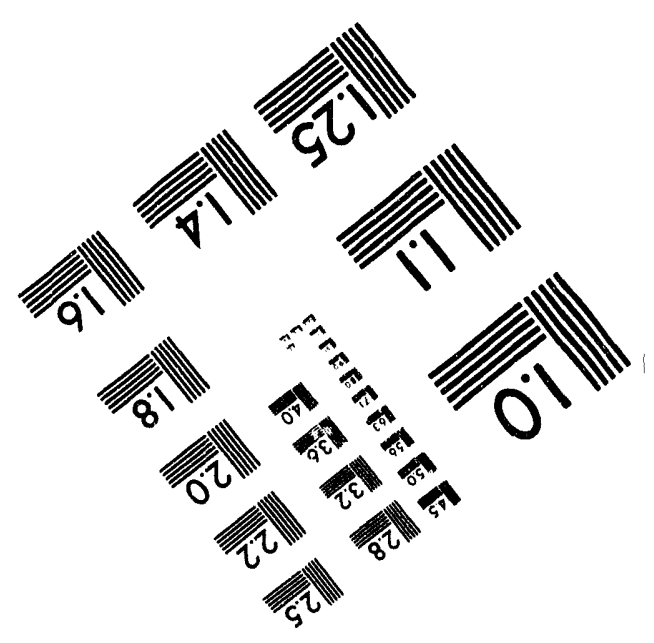





\title{
DOE/ER/12899-TI
}

\section{A Fundamental Study of \\ Contributon Transport Theory and \\ Channel Theory Applications}

Final Project Report

\author{
M.L. Williams
}

Nuclear Science Center

Louisiana State University

Baton Rouge, LA 70803-5820

July 1994

\begin{abstract}
Prepared for
THE U.S DEPARTMENT OF ENERGY

AGREEMENT NO. DE-FG07-89ER12899
\end{abstract}




\title{
A FUNDAMENTAL STUDY OF CONTRIBUTON TRANSPORT THEORY AND CHANNEL THEORY APPLICATIONS
}

\author{
M.L. Williams \\ Professor of Nuclear Engineering \\ Louisiana State University
}

\begin{abstract}
The objective of this project was to develop a new theoretical method called "contributon theory" that can be used to gain deeper understanding of radiation transport phenomena. The method had been proposed during the 1970's as a promising technique for improving radiation shield designs, and was actually applied to several reactor shielding studies at that time. However the theoretical basis of the method was never firmly established, and numerical procedures were not developed for efficient use of the method. Hence one important component of this project is to perform a study of the basic theory of contributons to understand how to better apply the method, and to develop calculational methods for using the theoretical tools effectively. Furthermore, the fundamrntal concepts of contributon theory can be applied in other areas besicies shielding optimization, including Monte Carlo calculations and neutron spectrum analysis; therefore the project also examined possible extended uses of contributon theory. Finally, in order for the method to become a useful tool for radiation transport analysis, it is necessary to demonstrate that it can be used to analyze realistic configurations; therefore the project also has developed computer programs that can be applied to realistic systems, and has performed analysis on several representative example problems, including a space-reactor mockup experiment, light water reactor pressure vessel dosimetry analysis, neutron transport calculations for the Hiroshima weapon radiation transport through air, and Monte Carlo analysis of an optically thick shield containing an irregular perturbation.
\end{abstract}




\section{BACKGROUND}

A major concern in radiation transport analysis is determining how radiation from some source contained in a complex medium is transferred through the system and registers "response" on a detection device. The particular set of particles responsible for generating the observed detector response is usually a small fraction of the overall population, and it generally has a very different behavior than the remaining set of particles that do not contribute to the response. However the entire response registered on the detector is carried by this special subset of the population. "Contributons" are defined to be pseudo-particles that carry response from the radiation source to the detector. If each contributon is defined to carry one unit of response, then the amount of potential response carried by radiation within any phase space volume is numerically equal to the number of contributons contained in the volume. It can be shown the contributon angular flux, which physically corresponds to the "angular response flux", obeys an equation that is very similar in form to the Boltzmann transport equation for a purely scattering medium. Manipulation of this equation leads to the "contributon conservation principle": contributons are emitted from the radiation source and can never be lost from the system until they arrive at the detector, where they are eliminated by observation. By examining the behavior of the contributon distribution, much can be learned about the radiation responsible for the observed detector response. This understanding may be is important for several reasons, including the fact that efficient placement of shielding material depends on knowing the spatial paths and energy distribution of the specific radiation contributing to the observed response. Another possible use of the contributon distribution is to correlate measured spectral data at the detector location to the radiation spectrum at some other location in the system.

\section{ACCOMPLISHMENTS}

A number of significant accomplishments were obtained as part of this study. Most of these have been previously documented in various publications, but are summarized below. For completeness, some of the most important publications are included in the appendix of this final report, and are referred to herein.

\section{Development of Theoretical Basis for Generalized Contributon Theory.}

An extensive work describing various elements of contributon theory and discussing many new properties of the contributon fields was completed. This work is labeled "Reference 1 " in the 
appendix. The Generalized Contributon Transport Equation was derived from the forward and adjoint Boltzmann equaxions, and several contributon variables were introduced, including the angular response density and flux, scalar response flux, response current, and response density. Expressions for "contributon scattering kernels" were derived. The contributon response conservation law was derived and its implications discussed. A lumped differential equation describing the time-dependant variation in the total response contained in a system was derived, and was used to verify the interpretation of the response density. This development also introduced expressions for the contributon mean lifetime, diffusion length, and average speed. These parameters can be used to provide a quantitative measure of the average distance and travel time required for a contributon to transfer response from the source to the detector. Spherical harmonic moments of the angular response flux were examined and related to the moments of the forward and adjoint fluxes, which allows computation of the contributon field variables directly from the moments computed in a transport calculation. These expressions complement those presented earlier in terms of angular flux variables.

\section{Spatial Channel Theory}

The contributon response variables were used to develop a technique called "spatial channel theory" that can be utilized to determine spatial transport paths followed through the system by the response. This method is useful in radiation shield design, because it provides guidance on effective location of shielding that can "plug" streaming channels followed by the particles responsible for the observed detector response. The contributon response density is shown to indicate spatial regions that contain high concentrations of potential response, and locations of high response location are usually effective locations for radiation shielding. It is shown that the response flow throughout is described by the contributon response current, a vector field. The properties of this vector field were studied in detail. The streamlines of the vector field indicate the direction of response flow, and will always originate at the source and terminate at the detector (sink). The streamlines are determined by iso-contours of a spacedependant variable called the stream function (which is analogous to the same quantity encountered in fluid mechanics), that can be calculated from the vector components of the response current. expressions. The response current is written in terms of a scalar and vector potential function. The scalar potential obeys a Poisson equation in which the source and sink terms correspond to the response source and detector locations. The idealized case of a "contributon dipole" (point source-point detector) is introduced, and it is shown that for this problem the scalar potential equation is actually independent of the system configuration, and can be easily computed. However the scalar potential alone is insufficient to uniquely determine the response flow field because the response current is "rotational"; ie, it generally includes a contribution from the vector potential that gives rise to a component that appears similar to the 
vorticity in fluid mechanics. Unlike the scalar potential, the vorticity is affected by the particular distribution and composition of the system components. The defined variables describing the response flow field, such as the stream function/streamlines, scalar potential, vorticity, and contributon diffucion length have been computed for several dipole cases, and were shown to give very useful information that aids in understanding radiation transport between a source and detector. Details of this work are included in reference one in the appendix. This area of contributon theory has great promise for interesting and productive future work.

\section{Contributon Monte Carlo}

Because contributon theory describes transport of response by only the particles responsible for generating a particular detector reading, the response transport equation is very attractive for improving the efficiency of Monte Carlo calculations. By tracking contributons instead of "real" particles, the Monte Carlo calculation focuses its effort on the response flow paths that contribute to the desired detector response. Since contributons are never lost from the system by absorption or leakage, in theory the contributon Monte Carlo calculation should be $100 \%$ efficient, and an analog simulation would yield a zero variance answer. However the response source in the contributon transport equation is a function of the detector adjoint function, and contributon particles interact with the medium via a scatter kernel that appears like an adjoint- biased scattering cross section. Unfortunately the true adjoint function for the problem is not known prior to a detailed transport calculation, which means that the precise solution of the contributon transport equation is not practical. It is shown in reference one that an approximate representation for the adjoint function can be used to generate the response source and contributon scatter kernels; however, this introduces additional terms in the response transport equation that can be interpreted as response absorption and multiplication reactions. A method was suggested to use two dimensional discrete ordinates calculations to obtain approximate adjoint functions to compute the required contributon source and interaction data for a three dimensional contributon Monte Carlo calculation. In regions where the two and three dimensional geometries differ, a "perturbation region" exists that may either deplete or enhance the contributon population through absorption or multiplication reactions. A variation of this approach was actually developed into a Monte Carlo program as a LSU doctoral dissertation, and has been applied to a difficult deep penetration transport calculation. The results were very impressive, giving a significant reduction in CPU time with no loss in accuracy. This thesis work has been submitted for publication in a technical journal. With additional development contributon Monte Carlo could become an effective calculation tool for radiation transport analysis. 


\section{Contributon Slowing Down Theory}

Another area of research supported by this grant is concerned with understanding how the energy spectrum of contributons changes as the response is transferred from the source to the detector. For example, although a detector at one location in the system may be mainly sensitive to low energy radiation, the particles that carry the response may initially be emitted from the source as high energy radiation. During the transport process, the contributons must slow down in energy to be detected. Furthermore, the energy variation of the various materials in the system will significantly impact the contributon energy distribution as response is transported through the material. Energy intervals where the macroscopic cross section goes through a minimum, such as at a cross section "window", provide effective mechanisms for response transport, due to the long mean-free path at these energies. The effect is that contributons tend to accumulate in certain favorable energy channels as they pass through the system. In reference two, contained in the appendix, contributon theory is used to develop methods for examining these coupled spaceenergy transport phenomena. Methods are developed to display the energy channels followed by the response flow so that at any point in the system it is possible to determine how much of the potential detector is distributed in energy. A new variable called the "response slowing down density" is defined to be the amount of response per unit time that slows down past any energy, as a function of spatial location. The response slowing down density, along with the three components of the response current, correspond to a four-dimensional vector field over the fourdimension space-lethargy continuum. Expressions for "space-lethargy streamlines" are presented to depict simultaneous contributon slowing down and space transport through one dimension geometries. The streamlines are obtained as the iso-contours of a space-lethargy stream function that is calculated from the slowing down density and current component. Contributon slowing down theory is applied to analysis of surveillance dosimetry placed in the cavity of a PWR, and to understanding how neutrons generated by the Little Boy device were transported through air to activate the cobalt in a steel structure approximately 1400 meters from the source. Results of these studies are presented in reference two in the appendix.

\section{Applications of Contributon Theory}

Several realistic applications of various aspects of contributon theory were considered in this project. Both references one and two in the appendix describe some of these applications; and as mentioned previously, several other applications have been addressed in the contributon Monte Carlo study. Some other applications that have not been described earlier are :

use of contributon theory to understand the transport paths followed by neutrons produced in the core of a power reactor in order to be detected on an ex-core monitor located with in the reactor cavity. This work has been performed by a LSU graduate student as thesis research. 
O use of contributon theory to determine the transport paths within an experiment mockup design for the NERVA space reactor. Results of this work were included in a technical presentation given at a conference on space reactors. A copy of the paper is provided as reference three in the appendix.

\section{GRADUATE STUDENT SUPPORT}

A significant fraction of the funds in this grant was used to support graduate student research assistantships. These students made important contributions to the overall success of the project. The following is a list of LSU graduate students who received support from this grant:

Mr. H. Manohara (MS thesis work)

Ms. S. Ghosn (MS thesis work)

Mr. C. Aboughantous (PhD dissertation work)

Reprints remove

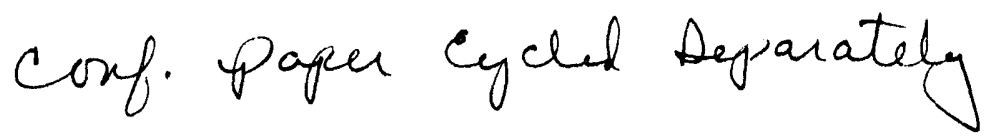



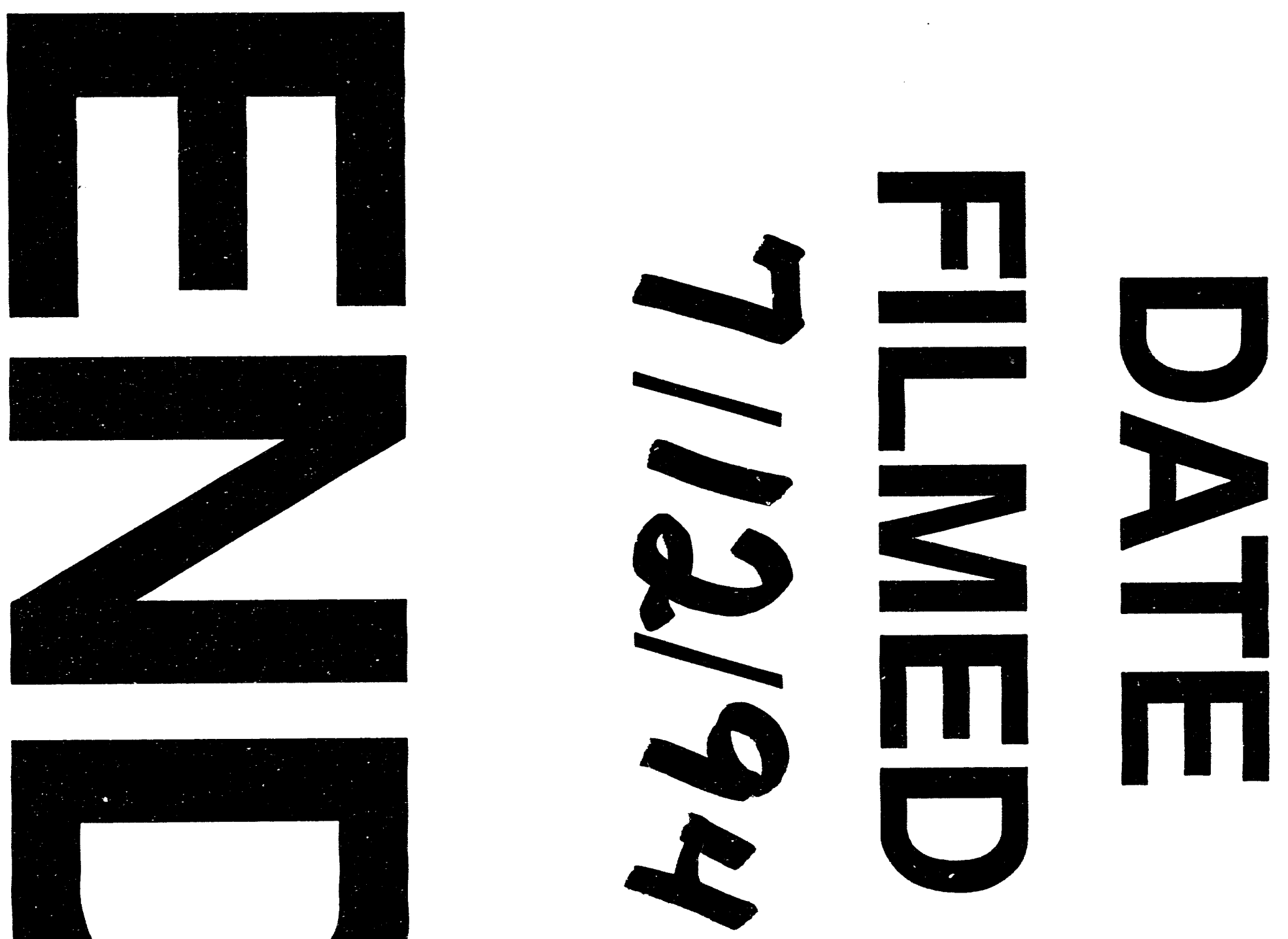

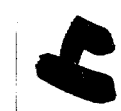

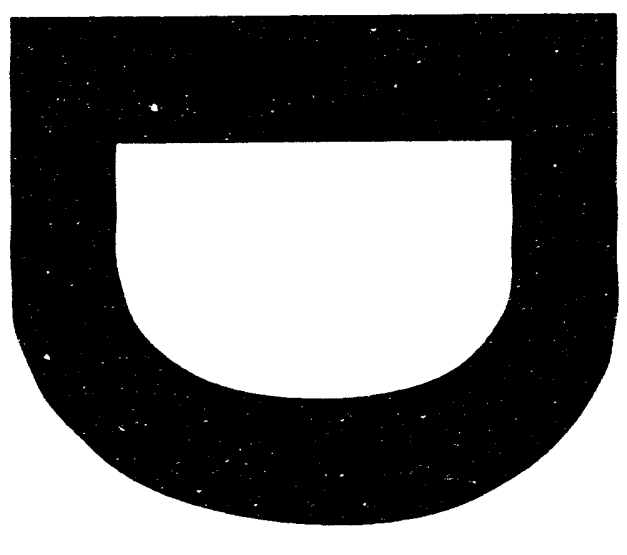




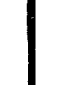

